\title{
NON-COMMUTATIVE EXTENSIONS OF CLASSICAL THEORIES IN PHYSICS
}

\author{
Richard Kerner \\ Laboratoire de Gravitation et Cosmologie Relativistes, \\ Université Pierre-et-Marie-Curie - CNRS ESA 7065, \\ Tour 22, 4-ème étage, Boîte 142 \\ 4, Place Jussieu, 75005 Paris, France \\ e-mail: rk@ccr.jussieu.fr
}

\begin{abstract}
We propose a short introductory overview of the non-commutative extensions of several classical physical theories. After a general discussion of the reasons that suggest that the non-commutativity is a major issue that will eventually lead to the unification of gravity with other fundamental interactions, we display examples of non-commutative generalizations of known geometries.

Finally we discuss the general properties of the algebras that could become generalizations of algebras of smooth functions on Minkowskian (Riemannian) manifolds, needed for the description of Quantum Gravity.
\end{abstract}




\section{Deformations of Space-Time and Phase space Geometries.}

The two most important branches of modern physics created in the beginning of this century, the General Relativity and Quantum Theory, possess their welldefined classical counterparts, the Newtonian gravity theory mechanics, which are obtained as limits of these theories when the parameters $c^{-1}$ or $\hbar$ The mathematical expression of this fact is formulated in terms of the deformations of the respective structures. The notion of deformation plays the central rôle in modern attempts which try to generalize the geometrical description of physical realm.

To be more precise, we can cite the example of the relation existing between the Lorentz and the Galilei groups: the Lorentz group can be considered as deformation of the Galilei group, with the characteristic parameter $c^{-1}$; when this parameter tends to zero, the Lorentz group is said to undergo the contraction into the Galilei group. Similarly, the quantization procedure proposed by J.E.Moyal [1] is a deformation of the usual Poisson algebra which is contracted back to it when the characteristic parameter of deformation which is here the Planck constant $h$ tends to zero. Finally, Special Relativity may be considered as a contraction of General Relativity when the characteristic parameter $G$ tends to zero (although some space-times different from the Minkowskian one can appear when the Ricci tensor is put to zero).

Now, with three fundamental constants of Nature, $h, G$ and $c^{-1}$ serving as deformation parameters, one can imagine seven different contractions of the hypothetical unified theory that would deserve the name of "Relativistic Quantum Gravity", and which is yet to be invented. The seven contractions correspond to the vanishing of:

a) one of the three parameters, i.e $h, G$, or $c^{-1}$ only;

b) two parameters at once, i.e. $(h$ and $G),\left(h\right.$ and $\left.c^{-1}\right)$, and $\left(G\right.$ and $\left.c^{-1}\right)$;

c) all the three parameters at once, i.e. $\left(h, G\right.$ and $\left.c^{-1}\right)$.

The following Table shows the relations between the corresponding theories, as well as their usual denominations (when we know them...). We did not take into account the fact that taking the double limits might be non-commutative, which cannot be excluded a priori and would have made our diagram even more complicated.

Two of the theories displayed here have not found their realization yet: the "Relativistic Quantum Gravity" and the "Non-Relativistic Quantum Gravity". It is not at all clear whether these hypothetical theories can be realized without introducing some new deformation parameter depending on a new physical constant, and whether this constant should be independent or related to the three fundamental constants $h, c$ and $G$ or not.

It is also amusing to note that our diagram is three-dimensional - is it just a coincidence that we happen to live in three space dimensions, too ? 


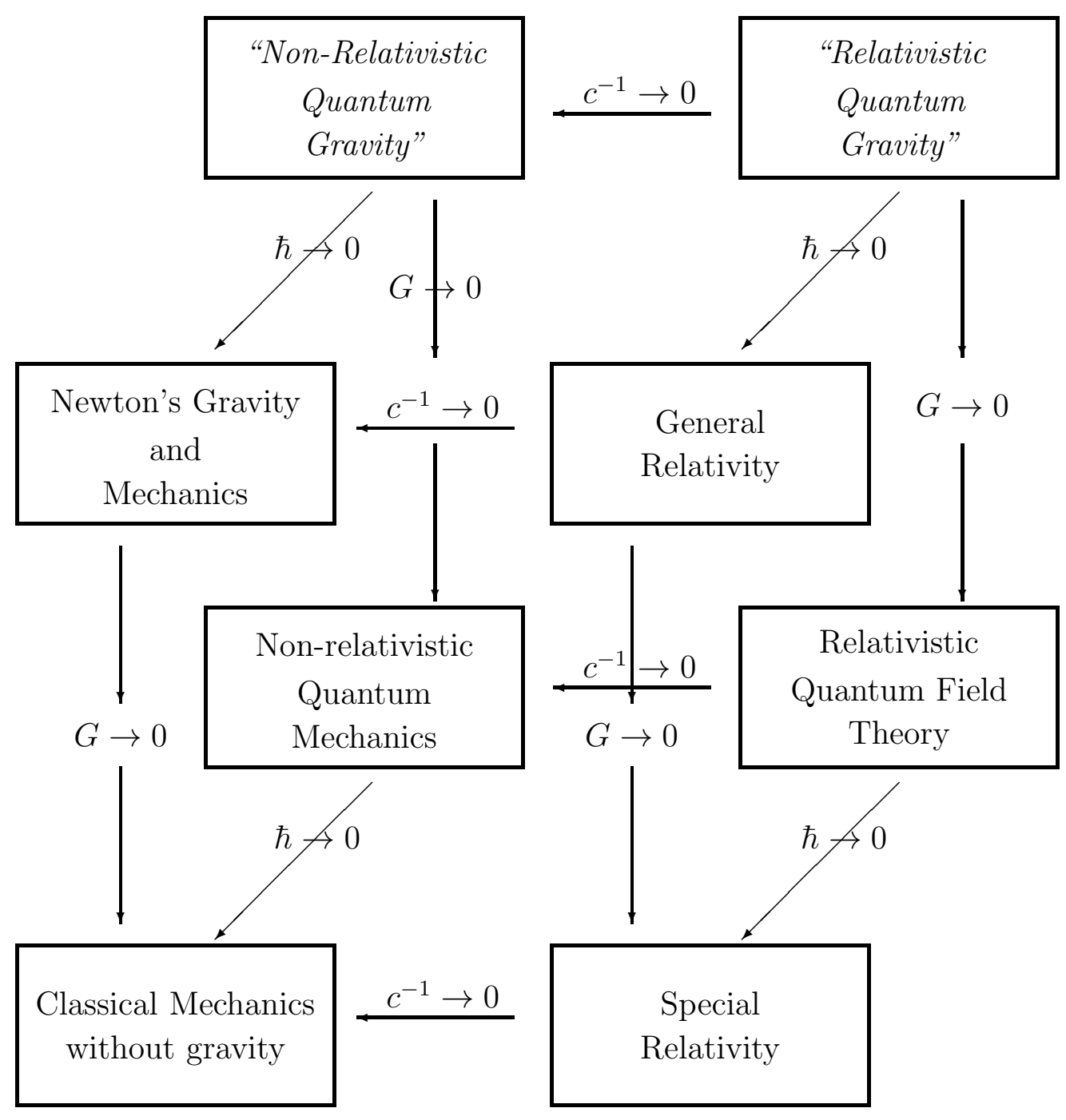

Eight limits of fundamental physical theories

Two limits (marked in italics) are still to be invented

In the above figure, the contractions (symbolized by the arrows coinciding with the edges of the cube) relate two-by-two different space-time or phase space geometries. The best way to describe a geometry is, in our sense, to define the set of variables (forming an algebra) that in a natural way would generalize the algebra of local coordinates in these spaces.

P.A.M.Dirac was already aware of the possibility of a radical modification of geometrical notions, and in his fundamental papers written in 1926 [2] he evokes the possibility of describing the phase space physics in terms of a noncommutative analogue of the algebra of functions, which he referred to as the "quantum algebra", together with its derivations, which he called "quantum differentiations". 
Of course, this kind of geometry seemed strange and even useless from the point of view of General Relativity. Einstein thought that further problems of physics should be solved by subsequent development of geometrical ideas, and it seemed to him that to have $a \times b$ not equal to $b \times a$ was something that does not fit very well with geometry as he understood it [3]

During several decades, mostly in the sixties and the seventies, a lot of efforts have been made in order to find a unifying approach to both these great theories.In doing so, people either tried to generalize one of the two theories so that the other one would follow, or tried to merge them together via embedding into some more general unified theory. Most of the activities in this field rather belonged to the first category.

The Hamiltonian formulation of General Relativity by R.Arnowitt, S.Deser and C.Misner [4], and later the Wheeler-De Witt equation which generalizes Schrödinger's equation for quantum wave functions describing the state of a 3dimensional geometry of the Universe [5] can be considered as a first attempt to quantize the General Relativity. The geometric quantization developed by J.M.Souriau, D.Simms, and B.Kostant ([6], [7], [8]) tried to derive the rules of quantum mechanics by interpreting the observables and state vectors as elements of algebras of operators and functions defined on classical manifolds with sufficiently rich geometry, (e.g. symplectic manifolds, fibre bundles, jet spaces).

Simultaneous consideration of the two most important new physical theories of this century, the General Relativity and Quantum Mechanics, did not bring a common tool for the description of the nature of spacetime at the microscopic level. The General Relativity develops our knowledge about global properties of space and time at very large distances, and raises the questions concerning the global topology of the Universe.

The methods of Differential Geometry which are the best adapted as the mathematical language of this theory, are very different from the methods of Quantum Physics, in which one studies the properties of the algebra of observables, considering the state vectors, as well as geometric points and trajectories, as artefacts and secondary notions. This approach has been inspired by the works of John von Neumann [9], and has much in common with the noncommutative geometry, where the very notion of a point loses its meaning.

A strong flavor of non-commutativity is also present in A. Ashtekar's approach to quantum gravity, in which the notion of coordinates becomes secondary, the only intrinsic information being encoded in the loop space (see, e.g. in A. Ashtekar [10], or C. Rovelli [11])

In the next section, we shall give a few arguments in favor of the hypothesis that the realization of a theory taking into account quantum effects in gravitation should also lead to the abandon of usual notion of coordinates and differential manifolds and to the introduction of non-commutative extensions of algebras of smooth functions on manifolds. We shall also see that such algebras can act on free modules, which becomes a natural generalization of gauge theories described mathematically as connections and curvatures on fibre bundles. 


\section{Why the coordinates should not commute at Planck's scale.}

There are several well-known arguments which suggest that the dynamical interplay between Quantum Theory and Gravitation should lead to a noncommutative version of space-time. Let us recall the few ones that are cited most frequently:

* A semi-classical argument that involves black-hole creation at very small distances: as a matter of fact, if the General Relativity remains valid at the Planck scale, then any localization of events should become impossible at the

distances of the order of $\lambda_{P}=\sqrt{\frac{\hbar G}{c^{3}}}$. Indeed, according to quantum mechanical principles, lo localize an event in space-time within the radius $\Delta x^{\mu} \sim a$, one need to employ the energy of the order $a^{-1}$. When $a$ becomes too small, the creation of a mini black hole becomes possible, thus excluding from the observation that portion of the space-time and making further localization meaningless.

Therefore, the localization is possible only if we impose the following limitation on the time interval:

$$
\Delta x^{0}\left(\Sigma \Delta x^{k}\right) \geq \lambda_{P}^{2} \quad \text { and } \quad \Delta x^{k} \Delta x^{m} \geq \lambda_{P}^{2}
$$

in order to avoid the black hole creation at the microscopic level.

** The topology of the space-time should be sensitive to the states of the fields which are in presence - and vice versa, quantum evolution of any field, including gravity, should take into account all possible field configurations, also corresponding to the fields existing in space-times with radically different topologies (a creation of a black hole is but the simplest example; one should also take into account other "exotic" configurations, such as multiple EinsteinRosen bridges (the so-called "wormholes", leading in the limit of great $N$ to the space-time foam.

Now, as any quantum measurement process may also lead to topological modifications, again the coordinates of an event found before and after any measurement can no more be compared, because they might refer to uncompatible coordinate patches in different local maps. As a result, quantum measures of coordinates themselves become non-commutative, and the algebra of functions on the space-time, supposed to contain also all possible local coordinates, must be replaced by its non-commutative extension, better adapted to describe the space-time foam.

*** Since the coordinates $x^{\mu}$ are endowed with a length scale, the metric must enter at certain stage in order to measure it. After quantization, the components of the tensor $g_{\mu \nu}$ become a set of dynamical fields, whose behaviour is determined by the propagators and, at least at the lowest perturbative level, by two-point correlation functions. As any other field, the components of the metric tensor will display quantum fluctuations, making impossible precise measurements of distances, and therefore, any precise definition of coordinates. 
Our aim here is not to discuss all possible arguments suggesting that at the Planck scale not only the positions and momenta do not commute anymore, but also the coordinates themselves should belong to a non-commutative algebra. In what follows, we shall take it for granted that such is the case, and shall expose in a concise way, on the example of the simplest finite non-commutative algebra, which is the algebra of complex $n \times n$ matrices, how almost all the notions of usual differential geometry can be extended to the non-commutative case. We shall also show how the gauge theories and the analogs of the fibre bundle spaces and Kaluza-Klein geometries can be generalized in the non-commutative setting.

Finally, as our main subject is the hypothetical Quantum Gravity theory, and because it has to have also a limit as Relativistic Field theory when gravity is switched off, we shall analyze the conequences of the Poincaré invariance that must be imposed on any theory of this type.

\section{Non-commutative differential geometry}

In the examples of non-commutative generalizations of spaces of states or of algebras of observables, we have looked up to now only at the linear cases. A most general non-commutative geometry should imitate the situations encountered in the ordinary differential geometry of manifolds. Therefore, we should replace the algebra of smooth functions on a manifold, (the maximal ideals of this algebra can be identified as points of the corresponding manifold) by an more general associative algebra, which can be non-commutative. The derivations of this algebra will naturally generalize the notion of vector fields; their dual space will generalize the fields of 1-forms, and one can continue as far as possible, trying to construct the analogues of a metric, integration, volume element, Hodge duality, Lie derivatives, connection and curvature, and so forth. It is amazing how almost all of these objects known from the classical version of differential geometry find their counterparts in the non-commutative case.

The differential algebras of this type have been studied by A.Quillen [12], A.Connes [13] and M.Dubois-Violette [14]; their application to new mathematical models of the gauge theories, including the standard model of electroweak interactions of Weinberg and Salam, has been worked out by M.Dubois-Violette et al [15],[16], by A.Connes and J.Lott [17], R.Coquereaux et al [18], and many other authors since. Here we shall give the simplest example of realization of the non-commutative geometry proposed in [15],[16], realized with the algebra of complex $n \times n$ matrices, $M_{n}(\mathbf{C})$. Any element of $M_{n}(\mathbf{C})$ can be represented as a linear combination of the unit $n \times n$ matrix 1 and $\left(n^{2}-1\right)$ hermitian traceless matrices $E_{k}, \mathrm{k}=1,2, \ldots,\left(n^{2}-1\right)$ :

$$
B=\beta 1+\sum \alpha^{k} E_{k}
$$

One can choose the basis in which the following relations hold:

$$
E_{k} E_{m}=\left(\frac{1}{n}\right) g_{k m} 1+S_{k m}^{j} E_{j}-\left(\frac{i}{2}\right) C_{k m}^{j} E_{j}
$$


with real coefficients satisfying $S_{k m}^{j}=S_{m k}^{j}, S_{k m}^{k}=0, C_{k m}^{j}=-C_{m k}^{j}, C_{k m}^{k}=0$, and $g_{k m}=C_{k l}^{p} C_{p m}^{l}$. Then $C_{k l}^{m}$ are the structure constants of the Lie group $S L(n, C)$, and $g_{k l}$ its Killing-Cartan metric tensor. All the derivations of the algebra $M_{n}(C)$ are interior, i.e. the basis of the derivations is given by the operators $\partial_{k}$ such that

$$
\partial_{k} E_{m}=\operatorname{ad}\left(i E_{k}\right) E_{m}=i\left[E_{k}, E_{m}\right]=C_{k m}^{l} E_{l}
$$

By virtue of the Jacobi identity, we have

$$
\partial_{k} \partial_{m}-\partial_{m} \partial_{k}=C_{k m}^{l} \partial_{l}
$$

The linear space of derivations of $M_{n}(C)$, denoted by $\operatorname{Der}\left(M_{n}(C)\right.$, is not a left module over the algebra $M_{n}(C)$ - this is the first important difference with respect to the usual differential geometry, in which a vector field can be multiplied on the left by a function, producing a new vector field. The canonical basis of 1 -forms dual to the derivations $\partial_{k}$ is defined formally by the relations

$$
\theta^{k}\left(\partial_{m}\right)=\delta_{m}^{k} 1
$$

These 1 -forms span a left module over $M_{n}(C)$, i.e. $E_{l} \theta^{k}$ is also a well-defined 1-form; indeed, $E_{l} \theta^{k}\left(\partial_{m}\right)=E_{l} \delta_{m}^{k} 1=E_{l} \delta_{m}^{k}$

The exterior differential $d$ is defined as usual, first on the 0-forms ("functions") by the identity

$$
d f(X)=X f
$$

with $f$ a function, $X$ an arbitrary vector field. Here we have

$$
(d 1)\left(\partial_{m}\right)=\partial_{m} 1=\operatorname{ad}\left(i E_{m}\right) 1=i\left[E_{m}, 1\right]=0
$$

so that $d 1=0$, and

$$
d E_{k}\left(\partial_{m}\right)=\partial_{m}\left(E_{k}\right)=i\left[E_{k}, E_{m}\right]=C_{m k}^{l} E_{l}
$$

Because the Lie algebra $S L(n, C)$ is semi-simple, the matrices $C_{k m}^{l}$ are nondegenerate, and the above relation can be solved in $\theta^{k}$ 's giving the explicit expression

$$
d E_{k}=C_{k m}^{l} E_{l} \theta^{m}
$$

The fact that $d^{2}=0$ follows then directly from the Jacobi identity. The Grassmann algebra of p-forms is defined as usual, with the wedge product

$$
\theta^{k} \wedge \theta^{m}=\left(\frac{1}{2}\right)\left(\theta^{k} \otimes \theta^{m}-\theta^{m} \otimes \theta^{k}\right)
$$

We have then

$$
d \theta^{k}+\left(\frac{1}{2}\right) C_{m l}^{k} \theta^{m} \theta^{l}=0
$$


If we define the canonical 1-form $\theta=\sum E_{k} \theta^{k}$, we can easily prove that it is coordinate-independent. Moreover, it satisfies the important relation

$$
d \theta+\theta \wedge \theta=0
$$

Let $\omega$ be a $p$-form. The anti-derivation $i_{X}$ with respect to a vector field $X$ can be defined as usual,

$$
\left(i_{X} \omega\right)\left(X_{1}, X_{2}, \ldots, X_{p-1}\right)=\omega\left(X, X_{1}, X_{2}, \ldots, X_{p-1}\right)
$$

The Lie derivative of a $p$-form $\omega$ with respect to a vector field $X$ is defined as

$$
L_{X} \omega=\left(\begin{array}{lll}
i_{X} & d+d & i_{X}
\end{array}\right) \omega
$$

It is easy to check now that the 2 -form $\Omega=d \theta$ is invariant with respect to the derivations of $A$, i.e. that

$$
L_{X} \Omega=0
$$

for any vector field $X$ belonging to $\operatorname{Der}\left(M_{n}(C)\right)$. The 2 -form $\Omega$ is also nondegenerate, and it is a closed 2 -form by definition, because

$$
d \Omega=d^{2} \theta=0
$$

The 2 -form $\Omega$ defines a Hamiltonian structure in the algebra $M_{n}(C)$ in the following sense:

Let $f \in M_{n}(C)$ be an element of our algebra; then $\operatorname{Ham}_{f}$ is the Hamiltonian vector field of $f$ defined by the equality

$$
\Omega\left(\operatorname{Ham}_{f}, X\right)=X \quad f
$$

for any $X$ belonging to $\operatorname{Der}\left(M_{n}(C)\right)$ The Poisson bracket of two "functions" (observables) $f$ and $g$ is then defined as

$$
\{f, g\}=\Omega\left(\operatorname{Ham}_{f}, \operatorname{Ham}_{g}\right)
$$

A simple computation shows then that

$$
\left\{E_{k}, E_{m}\right\}=\Omega\left(\partial_{k}, \partial_{m}\right)=i\left[E_{k}, E_{m}\right]
$$

Therefore, in our simple version of non-commutative geometry, classical and quantum mechanics merge into one and the same structure: the Poisson bracket of any two matrix "functions" (observables) is equal, up to a factor that can be chosen as the Planck constant $h$, to their commutator .

This simple and beautiful picture is of course somewhat perturbed in the case of infinite-dimensional algebras for which not all the derivations are interior and might have other representations than the commutator with an observable.

The volume element induced by the canonical Cartan-Killing metric and the 
corresponding Hodge duality ^ can be also introduced in a classical manner. The volume element is given by

$$
\eta=\frac{1}{\left(n^{2}-1\right) !} \epsilon_{i_{1} i_{2} \ldots i_{n^{2}-1}} \theta^{i_{1}} \wedge \theta^{i_{2}} \wedge \ldots \wedge \theta^{i_{n^{2}-1}}
$$

Any $n^{2}-1$-form is proportional to the volume element $\eta$; the integral of such a form will be defined as the trace of the matrix coefficient in front of $\eta$. Then the scalar product is readily introduced for any couple of $p$-forms $\alpha$ and $\beta$ as follows:

$$
(\alpha, \beta)=\int(\alpha \wedge \star \beta)
$$

With this formalism we can generalize the notions of gauge fields if we use the non-commutative matrix algebra as the analogue of the algebra of functions defined on a vertical space of a principal fibre bundle. Then we will be able to compute lagrangian densities that may be used in the variational principle producing dynamical field equations.

We shall see in the next section how this formulation of gauge theories contains besides the $S U(n)$ gauge fields also scalar multiples in the adjoint representation, which have the rôle of the Higgs fields in standard electroweak theory.

\section{Non-commutative analog of Kaluza-Klein and gauge theories.}

At this stage we can introduce a non-commutative analogue of Kaluza-Klein type theory, which will lead to a generalization of gauge field theories. In ordinary differential geometry the fact of using a Cartesian product of two differential manifolds, or a fibre bundle locally diffeomorphic with such a product, can be translated into the language of the corresponding function algebras; as a matter of fact, in the case of the Cartesian product of two manifolds, the algebra of functions defined on it is the tensorial product of algebras of functions defined on each of the manifolds separately.

Consider the space-time manifold $V_{4}$ with its algebra of smooth functions $C^{\infty}\left(V_{4}\right)$. Let us define the tensor product

$$
A=C^{\infty}\left(V_{4}\right) \otimes M_{n}(C)
$$

It can be shown (cf.[13]) that

$$
\operatorname{Der}(A)=\left[\operatorname{Der}\left(C^{\infty}\left(V_{4}\right)\right) \otimes 1\right] \oplus\left[C^{\infty}\left(V_{4}\right) \otimes \operatorname{Der}\left(M_{n}(C)\right]\right.
$$

In other words, a general derivation in our tensor product algebra replacing the algebra of smooth functions on a fibre bundle space, can be written as the following vector field

$$
X=X^{\mu}(x) \partial_{\mu}+\xi^{k}(x) \partial_{k}
$$

with $\mu, \nu=0,1,2,3 ; k, l=1,2, \ldots,\left(n^{2}-1\right)$. A general 1-form defined on such vector fields splits naturally into four different components:

$$
A=A_{\mu}^{0}(x) 1 d x^{\mu}+A_{\mu}^{k}(x) E_{k} d x^{\mu}+B_{m}^{0}(x) 1 \theta^{m}+B_{m}^{k}(x) E_{k} \theta^{m}
$$


The exterior differential of a 1-form $A$ takes into account the two kinds of differentiation; e.g. for a general matrix-valued 0-form ("function") $\Phi=\Phi^{0} 1(x)+$ $\Phi^{m}(x) E_{m}$ we have

$$
d(\Phi)=\left(\partial_{\mu} \Phi^{0}\right) d x^{\mu}+\left(\partial_{\mu} \Phi^{m}\right) E_{m} d x^{\mu}+\Phi^{m} C_{k m}^{l} E_{l} \theta^{k}
$$

The notion of covariant derivation can be introduced quite naturally by considering a free (right) hermitian module $H$ over the algebra $A$. If we choose a unitary element $e$ in $H$, then any element of $H$ can be represented as $\Phi=e B$, with $B \in A$. Then the covariant derivative must have the following basic property:

$$
\nabla(\Phi D)=(\nabla \Phi) D+\Phi \otimes d D
$$

for arbitrary $\Phi \in H, D \in A$ Now, if $\Phi=e B$, we shall have

$$
\nabla \Phi=(\nabla e) B+e \otimes d B
$$

and there exists a unique element $\alpha \in \Lambda^{1}\left(M_{n}(C)\right)$ such that

$$
\nabla e=e \otimes d \alpha
$$

satisfying the hermiticity condition $\bar{\alpha}=-\alpha$. The elements $B$ and $\alpha$ are called the components of the field $\Phi$ and the connection $\nabla$ in the gauge $e$.

Let $U$ be a unitary matrix from the algebra $A$. Under a change of gauge

$$
e \longrightarrow e U
$$

the components $B$ and $\alpha$ transform as follows:

$$
B \longrightarrow U^{-1} B \quad \alpha \longrightarrow U^{-1} \alpha U+U^{-1} d U
$$

This is the analogue of the gauge theory in the non-commutative case. When applied to the connection 1 -form (denoted now $A$ instead of $\alpha$ ), these principles lead to the following expression of the gauge field tensor $F=d A+A \wedge A$ :

$F=\left(F_{\mu \nu}^{0} 1+G_{\mu \nu}^{k} E_{k}\right) d x^{\mu} \wedge d x^{\nu}+\left[\left(D_{\mu} B_{l}^{0}\right) 1+\left(D_{\mu} B_{l}^{m} E_{m}\right)\right] d x^{\mu} \wedge \theta^{l}+G_{k l}^{m} E_{m} \theta^{k} \wedge \theta^{l}$

where

$$
F_{\mu \nu}^{0}=\partial_{\mu} A_{\nu}^{0}-\partial_{\nu} A_{\mu}^{0}
$$

represents the abelian $U(1)$-gauge field;

$$
G_{\mu \nu}^{k}=\partial_{\mu} A_{\nu}^{k}-\partial_{\nu} A_{\mu}^{k}+C_{l m}^{k} A_{\mu}^{l} A_{\nu}^{m}
$$

represents the $S U(2)$-gauge field;

$$
D_{m u} B_{k}^{0}=\left(\frac{1}{m}\right)\left(\partial_{\mu} B_{k}^{0}\right)
$$


is the derivative of the scalar triplet $B_{k}^{0}$;

$$
D_{\mu} B_{k}^{m}=\left(\frac{1}{m}\right)\left(\partial_{\mu} B_{k}^{m}+C_{s r}^{m} A_{\mu}^{s} B_{k}^{r}\right)
$$

is the covariant derivative of the scalar (Higgs type) multiplet $B_{k}^{m}$; finally,

$$
G_{k l}^{m}=\left(\frac{1}{m^{2}}\right)\left(C_{k l}^{p} B_{p}^{m}-C_{s r}^{m} B_{k}^{s} B_{l}^{r}\right)
$$

represents the potential contribution of the Higgs multiplet.

Here $m$ is the dimensional parameter $\left(\operatorname{dim}[\mathrm{m}]=\mathrm{cm}^{-1}\right)$ introduced in order to give the proper dimension to the 1 -forms $\theta^{k}$. The parameter $m$ can be later related to the characteristic mass scale of the theory. The generalized action integral is equal - in conformity with the definition of integration on the algebra of $\mathrm{p}$-forms in the non-commutative case - to the trace of the integral over spacetime $V_{4}$ of the expression $F \wedge \star F$ :

$$
\operatorname{Tr} \int(F \wedge \star F) d^{4} x
$$

The multiplet of scalar fields $B_{l}^{m}$ plays here the rôle of the symmetry-breaking Higgs-Kibble field, whose quartic potential appearing in the last part of the action integrand possesses multiple local minima or maxima.

In this example, when all other fields are set equal to 0, there exist several configurations of $B_{l}^{m}$ corresponding to vacuum states representing different gauge orbits. Indeed, it is easy to see that $G_{k l}^{m}=0$ not only when $B_{l}^{m}=0$, but also for $B_{l}^{m}=\delta_{l}^{m}$. These two vacua can not be transformed one into another by means of a gauge transformation, which is a novel feature when compared with the known classical versions of gauge theory coupled with Higgs fields.

Although this generalization of gauge theory including a non-commutative sector of differential geometry contains naturally the gauge group $S U(2) \times U(1)$, the Higgs multiplet arising here does not have the usually required properties, i.e. it is not a doublet of complex scalar fields coupled in a different way to the left- and right-handed fermions; we have instead a tensor multiplet $B_{l}^{m}$ that admits 16 different vacuum configurations, most of them degenerate saddle points in the parameter space. Also the mass spectrum of bosons appearing in the theory is not satisfactory. Developing the bosonic fields of the model, $A_{\mu}^{0}, A_{\mu}^{k}$ and $B_{k}^{0}$, and linearizing the equations around the vacua given by $B_{l}^{m}=0$ or $B_{l}^{m}=\delta_{l}^{m}$ respectively, we obtain on the gauge orbit $B_{l}^{m}=0$ :

- masses of $A_{\mu}^{0}$ and $A_{\mu}^{k}$ equal zero,

- masses of $B_{l}^{0}$ and $B_{l}^{m}$ all equal to $\sqrt{n} m$;

whereas on the gauge orbit $B_{l}^{m}=\delta_{l}^{m}$ :

- the $U(1)$ gauge field $A^{0} \mu$ remains massless while the $S U(2)$ - gauge field acquires the mass $\sqrt{2 n} m$;

- the scalar multiplet $B_{m}^{0}$ acquires the mass $\sqrt{2} m$, and the Higgs multiplet itself, $B_{l}^{m}$ develops a mass spectrum with values $0, \sqrt{2} m$ and $2 \sqrt{2} m$. 
which makes this version of unified $S U(2) \times U(1)$ theory unrealistic.

More realistic versions of non-commutative gauge models, reproducing quite well all the properties of the electroweak interactions required by the experiment, have been proposed by A.Connes and M.Lott [17], R. Coquereaux et al. [18], by M.Dubois-Violette et al., [15], [16], and by J.Fröhlich et al., [19]. In all these models the non-commutative algebra of complex matrices is tensorized with a $Z_{2}$-graded algebra, which in simplest realisation can be conceived as algebra of $2 \times 2$ matrices that splits into two linear subspaces called "even" (corresponding to diagonal matrices) and "odd" (corresponding to the off-diagonal matrices), with respective grades being 0 and 1, which under matrix multiplication add up modulo 2. The exterior derivations change the grade of an element by 1 , and satisfy the graded Leibniz rule

$$
d(A B)=(d A) B+(-1)^{\operatorname{grad}(A) \operatorname{grad}(B)} A d B
$$

This enables one to represent the connection form (interpreted as the gauge-field potential) in the following form:

$$
\left(\begin{array}{cc}
A & W^{+} \\
W^{-} & Z
\end{array}\right)
$$

where the gauge fields $A$ and $Z$ belong to the even part of the algebra, while the fields $W^{+}$and $W^{-}$belong to the odd part; moreover, all these fields are themselves $2 \times 2$ matrix-valued 1-forms. Developing this theory around the appropriately chosen vacuum configuration one can quite correctly reproduce the mass spectrum, with the mass of neutral $Z$-boson $\frac{2}{\sqrt{3}}$ times bigger than the mass of the charged $W$ - boson, which corresponds to the Weinberg angle of $30^{\circ}$. More details can be found in the papers cited above.

At this point one may try to imagine what a non-commutative extension of the General Relativity could look like ? Since a long time there exist many approaches in which the General Relativity was considered as a gauge theory, with gauge group being the infinite-dimensional group of diffeomorphisms of four-dimensional Riemannian manifolds. However, with the gauge group of this size little could be done in matter of computation and prediction, especially on the quantum level.

A more realistic direction consists in exploring the properties of linear approximation of a more complicated final version of the theory. Recently, J. Madore et al. in [20] have introduced the generalization of linear connections on matrix algebras defined above. With the usual definition of covariant derivation acting on the moving frame:

$$
D \theta^{\alpha}=-\omega_{\beta}^{\alpha} \otimes \theta^{\beta}
$$

Because the definition of covariant derivative requires that

$$
D(f \xi)=d f \otimes \xi+f D \xi
$$


the covariant derivative of an arbitrary 1 -form $\xi_{\alpha} \theta^{\alpha}$ is

$$
D\left(\xi_{\alpha} \theta^{\alpha}\right)=d \xi_{\alpha} \otimes \theta^{\alpha}-\xi_{\alpha} \omega_{\beta}^{\alpha} \theta^{\beta}
$$

The covariant derivative along a vector field $X$ is defined as

$$
D_{X} \xi=i_{X}(D \xi)
$$

and defines a mapping of $\Omega^{1}(V)$ on itself.

If the torsion vanishes, then one finds that

$$
D^{2} \theta^{\alpha}=-\Omega_{\beta}^{\alpha} \theta^{\beta}
$$

where $\Omega_{\beta}^{\alpha}=R_{\beta \gamma \delta}^{\alpha} \theta^{\gamma} \wedge \theta^{\delta}$ is the curvature 2-form.

The generalization of these formalism for the non-commutative case is quite obvious. We must replace the linear space of 1 -forms which span the tensor and the exterior algebras by the corresponding right $\mathcal{A}$-module of 1 -forms defined over our matrix algebra $\Omega^{1}\left(M_{n} \mathbf{C}\right)$ ). In the basis introduced in the previous section, $\theta^{k}, k=1,2, \ldots\left(n^{2}-1\right)$, we had

$$
d \theta^{k}=-\frac{1}{2} C_{l m}^{k} \theta^{l} \theta^{m}, \quad \text { and } \quad d f=[\theta, f] .
$$

It is easy to define the linear connection with vanishing torsion:

$$
D \theta^{r}=-\omega_{s}^{r} \otimes \theta^{s}, \quad \text { with } \quad \omega_{s}^{r}=-\frac{1}{2} C_{s t}^{r} \theta^{t}
$$

Introducing the permutation operator $\sigma$ as

$$
\sigma\left(\theta^{k} \otimes \theta^{m}\right)=\theta^{m} \otimes \theta^{k}
$$

we can express the commutativity of the algebra $\mathcal{C}^{\infty}\left(M_{4}\right)$

$$
D(\xi f)=D(f \xi)
$$

by writing

$$
D(\xi f)=\sigma(\xi \otimes d f)+(D \xi) f .
$$

The last condition can be maintained in a more general case as the requirement imposed on the connection 1-forms. It follows then that in the case of matrix algebras considered here, one has

$$
D\left(\left[f, \theta^{k}\right]\right)=\left[f, D \theta^{k}\right]=0,
$$

so that all the coefficients $\omega_{l m}^{k}$ must be in the center of $M_{n}(\mathbf{C})$, i.e. they are just complex numbers, and the torsionless connection defined above becomes unique.

The metric in the space of 1-forms over $M_{n}(\mathbf{C})$ has been already introduced as $g\left(\theta^{k} \otimes \theta^{m}\right)=g^{k m} \in \mathbf{C}$. The fact that $\omega_{(l m)}^{k}=0$ can be interpreted as 
the metricity of this connection. This leads to the unique definition of the corresponding curvature tensor:

$$
\Omega_{l m n}^{k}=\frac{1}{8} C_{l r}^{k} C_{m n}^{r}
$$

These constructions have been used already in [15] and [16], and can serve as the non-commutative extension of connexion and curvature on the tensor product of algebras $\mathcal{C}^{\infty}\left(M_{4}\right) \otimes M_{n}(\mathbf{C})$.

However, the fact that all geometrically important quantities like metric, connection and curvature coefficients, are forced to belong to the center of the non-commutative sector make the above generalization quite trivial and therefore unsatisfactory.

\section{Minkowskian space-time as a commutative limit}

In this section we shall discuss an important feature of any non-commutative geometry that contains the algebra of smooth functions on Minkowskian spacetime and is supposed to be Poincaré-invariant at least in the first orders of the deformation parameter. This result has been published in 1998 (M. DuboisViolette, J. Madore, R. Kerner, [21]). Similar ideas have been independently developed earlier by S. Doplicher, K. Friedenhagen and J.E. Roberts (cf. [22]).

The main idea is as follows. Suppose that the non-commutative geometry that is supposed to describe in an adequate way the quantum version of General Relativity contains in its center the infinite algebra of smooth functions on Minkowskian space-time. This infinite algebra serves as a representation space for the infinite-dimensional representation of the Poincaré group, in particular, the abelian group of translations, in the limit when the gravitational interaction becomes negligible, which shall correspond to the limit $\kappa \rightarrow 0$, where $\kappa$ is proportional to the gravitational coupling constant $G$.

It seems natural to suppose that the Poincaré invariance remains still valid before the limit is attained, at least in the linear approximation with respect to the deformation parameter $\kappa$. Then an important question to be answered appears, namely, what is the dimension of the non-commutative part of the full algebra before the limit is attained? As it is shown in the reference [21], it must be infinite-dimensional. In other words, it is impossible to impose the full Poincaré invariance on a tensor product of $\mathcal{C}^{\infty}\left(M_{4}\right)$ with a finite non-commutative algebra, as in the example with the matrix algebras considered in previous sections. These examples can be considered only as approximations to the correct theory of non-commutative space-time and gauge field theories.

Let us consider then a one-parameter family of associative algebras, $\mathcal{A}_{\kappa}$, whose limit at $\kappa=O$, denoted by $\mathcal{A}_{0}$, admits a well-defined action of the Poincaré group on it. When $\kappa \rightarrow 0$, one should attain as a classical limit certain algebra, obviously containing $\mathcal{C}^{\infty}\left(M_{4}\right)$, the algebra of smooth functions on the Minkowskian manifold:

$$
\mathcal{A}_{\kappa} \rightarrow \mathcal{A}_{0} \supset \mathcal{C}^{\infty}\left(M_{4}\right)
$$


The one-parameter family of associative algebras, $\mathcal{A}_{\kappa}$, can be analyzed with the help of the deformation theory developed in the well-known article by $\mathrm{F}$. Bayen, M. Flato, C. Fronsdal and A. Lichnerowicz (cf. [23]). It is supposed that all $\mathcal{A}_{\kappa}$ coincide - as vector spaces - with a fixed vector space $E$. The product of any two elements $f, g$ in $\mathcal{A}_{\kappa}$ can be expanded as follows:

$$
(f g)_{\kappa}=f g+\kappa c(f, g)+o\left(\kappa^{2}\right)
$$

where $f g=(f g)_{0}$ is the product in $\mathcal{A}_{0}$. We also assume that there is a common unit element 1 for all $\mathcal{A}_{\kappa}$. The commutators of any two elements $f, g$ in $\mathcal{A}_{\kappa}$ and in $\mathcal{A}_{O}$ are related via the following equation:

$$
[f, g]_{\kappa}=[f, g]_{0}-i \kappa\{f, g\}+o\left(\kappa^{2}\right)
$$

where $\{f, g\}=i(c(f, g)-c(g, f))$. The mapping $(f, g) \rightarrow c(f, g)$ is called a normalized Hochschild 2-cocycle of $\mathcal{A}_{0}$ with values in $\mathcal{A}_{0}$.

The derivation property of the commutator in $\mathcal{A}_{\kappa}$ should be maintained, which means that

$$
\left[h,(f g)_{\kappa}\right]_{\kappa}=\left([h, f]_{\kappa}, g\right)_{\kappa}+\left(f,[h, g]_{\kappa}\right)_{\kappa}
$$

Then, in the first order in $\kappa$, we get

$$
i([h, c(f, g)]-c([h, f], g)-c(f,[h, g]))=f\{h, g\}-\{h, f g\}+\{h, f\} g
$$

This implies that if $h \in \mathcal{Z}\left(\mathcal{A}_{0}\right)$, the center of the algebra $\mathcal{A}_{0}$, then the endomorphism $\delta_{h}: \delta_{h}(f)=\{h, f\}$ is a derivation of $\mathcal{A}_{0}$ :

$$
\{h,\{f, g\}\}=\{\{h, f\}, g\}+\{f,\{h, g\}\}
$$

The center of the algebra $\mathcal{A}_{0}$, denoted by $\mathcal{Z}(\mathcal{A})$, is stable under these derivations, and therefore, it closes under the bracket $\{$,$\} . This means that the Jacobi$ identity valid in all associative algebras $\mathcal{A}_{\kappa}$ remains valid, at least up to the second order in $\kappa$, in $\mathcal{A}_{0}$ :

$$
\begin{aligned}
\text { from } & {\left[f,[g, h]_{\kappa}\right]_{\kappa}+\left[g,[h, f]_{\kappa}\right]_{\kappa}+\left[h,[f, g]_{\kappa}\right]_{\kappa}=0 \quad \text { it follows } } \\
& \left\{f,\{g, h\}_{\kappa}\right\}_{\kappa}+\left\{g,\{h, f\}_{\kappa}\right\}_{\kappa}+\left\{h,\{f, g\}_{\kappa}\right\}_{\kappa}=0
\end{aligned}
$$

Summarizung up, we can make the following statement:

The center of $\mathcal{A}_{0}, \mathcal{Z}\left(\mathcal{A}_{0}\right)$, is a commutative Poisson algebra with the Poisson bracket given by

$$
i(c(f, g)-c(g, f))
$$

The linear mapping $z \rightarrow \delta_{z}$ maps $\mathcal{Z}\left(\mathcal{A}_{0}\right)$ into the Lie agebra of derivations of $\mathcal{A}_{0}: \quad \delta_{z}(f)=\{z, f\}, \quad$ for $z, f \in \mathcal{A}_{0}$

We wish to represent the non-commutative analog of real functions by Hermitian elements of the extended algebra of functions. Therefore, we should impose 
the following reality condition :

- all the $\mathcal{A}_{\kappa}$ are complex *-algebras, whose involutive vector spaces coincide with the unique space $E$;

- for any $f \in E$, also $f^{*} \in E$; moreover, we assume that there exists a unique hermitian element which is the common unit for all these algebras, $\mathbf{1}^{*}=\mathbf{1}$, such that

$$
(f g)_{\kappa}^{*}=\left(f^{*} g^{*}\right)_{\kappa}, \quad \text { and } \quad(\mathbf{1} f)_{\kappa}=(f \mathbf{1})_{\kappa}=f
$$

It follows that the normalized co-cycle $c(f, g)$ satisfies natural condition

$$
(c(f, g))^{*}=c\left(g^{*}, f^{*}\right)
$$

Thus, the set $\mathcal{Z}_{R}\left(\mathcal{A}_{0}\right.$ of all Hermitian elements of $\mathcal{Z}\left(\mathcal{A}_{0}\right.$ forms naturally a real Poisson algebra, and $z \rightarrow \delta_{z}$ maps it into the real Lie algebra $\operatorname{Der}\left(\mathcal{A}_{0}\right.$ of all Hermitian derivations of $\mathcal{A}_{0}$.

Now comes the main point: the necessary realization of the Poincaré invariance on these algebras. The family $\mathcal{A}_{\kappa}$ represents non-commutative extensions of the algebra of smooth functions on space-time. Even if these algebras are not Poincaré-invariant, we wish to recover the Poincaré-invariant physics on the usual Minkowski space in the limit when $\kappa \rightarrow 0$. Therefore, we must assume that the Poincaré group $\mathcal{P}$ acts via ${ }^{*}$-automorphisms on the limit algebra $\mathcal{A}_{0}$ :

$$
(\Lambda, a) \rightarrow D_{\Lambda, a)} \in \mathcal{L}\left(\mathcal{A}_{0}, \mathcal{A}_{0}\right)
$$

for any element $(\Lambda, a) \in \mathcal{P}$.

By hypothesis, the algebra $\mathcal{A}_{0}$ contains a ${ }^{*}$-subalgebra identified with the commutative algebra of smooth functions on Minkowski space, $\mathcal{C}^{\infty}\left(M_{4}\right)$. The action of $\mathcal{P}$ on $\mathcal{A}_{0}$ should induce the usual action of $\mathcal{P}$ on $\mathcal{C}^{\infty}\left(M_{4}\right)$ associated with the corresponding linear transformations in $M_{4}$.

We shall now argue that $\mathcal{C}^{\infty}\left(M_{4}\right)$ can not be the whole $\mathcal{A}_{0}$.

Indeed, suppose that $\mathcal{A}_{0}=\mathcal{C}^{\infty}\left(M_{4}\right)$. The, in view of the our previous satement concerning the Poisson structures, there exists a Poisson bracket on $M_{4}$. This Poisson bracket must be non-trivial, since we assumed that the $\mathcal{A}_{\kappa}$ are all non-commutative.

On the other hand, we know that there does not exist a non-trivial Poincaré invariant bracket on $M_{4}$. Indeed, let $(f, g) \rightarrow\{f, g\}$ be such a bracket. Then, in a given coordinate patch, it can be represented analytically as

$$
\{f, g\}=\Omega^{\mu \nu} \partial_{\mu} f \partial_{\nu} g
$$

whare $\Omega^{\mu \nu}=\left\{x^{\mu}, x^{\nu}\right\}$ must be an anti-symmetric tensor field on $M_{4}$, which is constant with respect to translations and Lorentz covariant.

However, the rotational invariance already implies that the three-vectors

$$
E^{i}=\Omega^{0 i} \quad \text { and } \quad B^{k}=\epsilon_{l m}^{k} \Omega^{l m}, \quad(i, k, l=1,2,3)
$$


should vanish, which means that $\Omega^{\mu \nu}=0$, and therefore, also $\{f, g\}=0$ for all $f, g \in \mathcal{C}^{\infty}\left(M_{4}\right)$.

It seems unreasonable to suppose that the Poincaré invariance is broken at the first order in $\kappa$, because at this order we expect to recover a spin-2 Poincaréinvariant theory, coupled to other physical fields. So, if the Poincaré invariance holds at the first order in $\kappa$, it follows that the inclusion $\mathcal{C}^{\infty}\left(M_{4}\right) \subset \mathcal{A}_{0}$ must be a strict one, i.e. the limit $\kappa \rightarrow 0$ of $\mathcal{A}_{\kappa}$ must contain an extra factor besides $\mathcal{C}^{\infty}\left(M_{4}\right)$. Therefore, the normalized two-cocycle $c($,$) of \mathcal{A}_{0}$ defined by

$$
(f g)_{\kappa}=f g+\kappa c(f, g)+o\left(\kappa^{2}\right)
$$

is supposed to be Poincaré-invariant, i.e. it has the property:

$$
\alpha_{(\Lambda, a)}(c(f, g))=c\left(\alpha_{(\Lambda, a)}(f), \alpha_{(\Lambda, a)}(g)\right)
$$

which implies the invariance of the $\kappa$-bracket:

$$
[f, g]_{\kappa}=[f, g]-i\{f, g\}+o\left(\kappa^{2}\right)
$$

Let us consider now the elements of $\mathcal{A}_{0}$ that belong to $\mathcal{C}^{\infty}\left(M_{4}\right)$ and generate the commutative algebra of smooth functions on $M_{4}: x^{\mu} \in \mathcal{C}^{\infty}\left(M_{4}\right)$. By definition, we have then

$$
\alpha_{(\Lambda, a)} x^{\mu}=\Lambda_{\nu}^{-1 \mu}\left(x^{\nu}-1 a^{\nu}\right)
$$

By choosing the origin, one can identify $\mathcal{C}^{\infty}\left(M_{4}\right)$ with the Hopf algebra of functions on the group of translations of $M_{4}$. Since $\mathcal{C}^{\infty}\left(M_{4}\right)$ is a subalgebra of $\mathcal{A}_{0}$, the algebra $\mathcal{A}_{O}$ is a bimodule over $\mathcal{C}^{\infty}\left(M_{4}\right)$. As a left $\mathcal{C}^{\infty}\left(M_{4}\right)$-module, $\mathcal{A}_{0}$ is isomorphic with the tensor product $\mathcal{C}^{\infty}\left(M_{4}\right) \otimes \mathcal{A}_{0}^{I}$, where $\mathcal{A}_{0}^{I}$ denotes the subalgebra of transitionally invariant elements of $\mathcal{A}_{0}$ :

$$
\mathcal{A}_{0}^{I}=\left\{f \in \mathcal{A}_{0} \mid \alpha_{(1, a)}(f)=f \text { for all } a\right\}
$$

In fact, $\mathcal{A}_{0}$ is isomorphic with $\mathcal{C}^{\infty}\left(M_{4}\right) \otimes \mathcal{A}_{0}^{I}$ as a $\left(\mathcal{C}^{\infty}\left(M_{4}\right), \mathcal{A}_{0}^{I}\right)$-bimodule. Thus in order to recover the complete algebraic structure of $\mathcal{A}_{0}$, it is sufficient to describe the right multiplication by elements of $\mathcal{C}^{\infty}\left(M_{4}\right)$ of the elements of $\mathcal{A}_{0}^{I}$. The algebra $\mathcal{A}_{0}^{I}$ is stable under the derivations induced by the generators of local coordinate variables $x^{\mu}$ :

$$
f \rightarrow \operatorname{ad}\left(x^{\mu}\right)(f)=\left[x^{\mu}, f\right]
$$

Therefore, for any $f \in \mathcal{A}_{0}^{I}$ one has

$$
f x^{\mu}=x^{\mu} f-a d\left(x^{\mu}\right)(f)
$$

or, in the tensorial representation $\mathcal{A}_{0}=\mathcal{C}^{\infty}\left(M_{4}\right) \otimes \mathcal{A}_{0}^{I}$ :

$$
f x^{\mu}=x^{\mu} \otimes f-\mathbf{1} \otimes \operatorname{ad}\left(x^{\mu}\right)(f) \text { for any } f \in \mathcal{A}_{0}^{I}
$$


From this we can deduce the right multiplication of $\mathcal{C}^{\infty}\left(M_{4}\right) \otimes \mathcal{A}_{0}^{I}$ by the elements of $\mathcal{C}^{\infty}\left(M_{4}\right)$. Let us denote by $X^{\mu}$ the four commuting derivations of $\mathcal{A}_{0}^{I}$ induced by $\operatorname{ad}\left(x^{\mu}\right)$. The algebra $\mathcal{A}_{0}^{I}$ is invariant under the action of the diffeomorphisms $\alpha_{(\Lambda, 0)}$.

Let us denote by $\alpha_{\Lambda}^{I}$ the homomorphism of the Lirentz group into the group $\operatorname{Aut}\left(\mathcal{A}_{0}^{I}\right)$ of all the ${ }^{*}$-automorphisms of $\mathcal{A}_{0}^{I}$.

Then one can summarize the above discussion of properties of our algebra by the following presentation of $\mathcal{A}_{0}$ :

We start with a unital ${ }^{*}$-algebra $\mathcal{A}_{0}^{I}$ equipped with four commuting antiHermitian derivations $X^{\mu}$ and the action $\Lambda \rightarrow \alpha_{\Lambda}^{I}$ of the Lorentz group through the automorphisms of $\mathcal{A}_{0}^{I}$ :

$$
\alpha_{\Lambda}^{I} \circ X^{\mu}=\Lambda^{-1}{ }_{\nu}^{\mu} X^{\nu} \circ \alpha_{\Lambda}^{I}
$$

The entire algebra $\mathcal{A}_{0}$ is generated as a unital *-algebra by $\mathcal{A}_{0}^{I}$ and the four Hermitian elements $x^{\mu}$ which satisfy the relations:

$$
\begin{gathered}
x^{\mu} x^{\nu}=x^{\nu} x^{\mu} \\
\text { and } \quad x^{\mu} f=f x^{\mu}+X^{\mu}(f) \quad \text { if } \quad f \in \mathcal{A}_{0}^{I}
\end{gathered}
$$

The Poincaré group acts on $\mathcal{A}_{0}$ as follows:

- for $x^{\mu} \in \mathcal{C}^{\infty}\left(M_{4}\right)$ :

$$
\alpha_{(\Lambda, a)}\left(x^{\mu}\right)=\Lambda_{\nu}^{-1 \mu}\left(x^{\nu}-a^{\nu} \mathbf{1}\right) ;
$$

- for $f \in \mathcal{A}_{0}^{I}$ :

$$
\alpha_{(\Lambda, a)}(f)=\alpha_{\Lambda}^{I}(f) .
$$

But we have assumed before that the bracket

$$
\{f, g\}=i(c(f, g)-c(g, f))
$$

does not vanish identically on $\mathcal{C}^{\infty}\left(M_{4}\right)$. This implies that the functions $c^{\mu \nu}$ defined as

$$
c^{\mu \nu}=c\left(x^{\mu}, x^{\nu}\right)
$$

do not all vanish. On the other hand, these functions being Lorentz covariant must belong to $\mathcal{A}_{0}^{I}$, so that we have

$$
\alpha_{\Lambda, a)}\left(c^{\mu \nu}\right)=\tilde{c}^{\mu \nu}
$$

and one has

$$
\alpha_{\Lambda}^{I}\left(c^{\mu \nu}\right)=\Lambda_{\rho}^{-1 \mu} \Lambda_{\sigma}^{-1 \nu} c^{\rho \sigma},
$$

so that the homomorphism of the Lorentz group into the group $\operatorname{Aut}\left(\mathcal{A}_{0}^{I}\right.$ of the *-automorphisms of $\mathcal{A}_{0}^{I}$ is never trivial.

This implies in turn that $\mathcal{A}_{0}^{I}$ cannot be a finite-dimensional algebra (like e.g. the complex matrix algebra discussed in our previous example), because 
on such an algebra all automorphisms are inner, and on the other hand, it is known that the Lorentz group has no non-trivial, finite dimensional unitary representations. Therefore, the extra factor that is present in $\mathcal{A}_{0}$ besides the usual infinite-dimensional algebra of functions (coordinates) on $M_{4}$ must be also infinite dimensional.

In view of previous analysis, the algebra $\mathcal{A}_{0}$ is the tensor product $\mathcal{C}^{\infty}\left(M_{4}\right) \otimes$ $\mathcal{A}_{O}^{I}$, with the Lorentz group acting via automorphisms on $\mathcal{A}_{0}^{I}$. Since the brackets $\left\{x^{\mu}, x^{\nu}\right\} \in \mathcal{A}_{0}^{I}$, the algebra $\mathcal{A}_{0}^{I}$ must contain as a subalgebra an algebra of functions on the union of Lorentz orbits of anti-symmetric 2-tensors. The coordinates on this algebra viewed as a manifold are just the brackets $\left\{x^{\mu}, x^{\nu}\right\}$. The orbits may be labeled by the following two parameters:

$$
\alpha=g_{\mu \rho} g_{\nu \lambda}\left\{x^{\mu}, x^{\rho}\right\}\left\{x^{\nu}, x^{\lambda}\right\} \quad \text { and } \quad \beta=\epsilon_{\mu \nu \rho \sigma}\left\{x^{\mu}, x^{\nu}\right\}\left\{x^{\rho}, x^{\sigma}\right\} .
$$

If we want to include the definitions of time reversal and parity, we should assume that whenever a given orbit $(\alpha, \beta)$ appears in the algebra, the orbit corresponding to $(\alpha,-\beta)$ should appear as well. When one has also $\left\{x^{\mu},\left\{x^{\nu}, x^{\lambda}\right\}\right\}=0$ for all values of indeces $\mu, \nu, \lambda$, then $\mathcal{A}_{0}^{I}$ is equal to the above algebra.

The simplest situation occurs when $\mathcal{C}^{\infty}\left(M_{4}\right)$ belongs to the center of $\mathcal{A}_{0}$. In this case the cocycle $c$ is antisymmetric (up to a co-boundary) on $\mathcal{C}^{\infty}\left(M_{4}\right)$, and also on the center $\mathcal{Z}\left(\mathcal{A}_{0}\right)$ itself. Then $\mathcal{A}_{0}$ is a commutative Poisson algebra, and the family $\mathcal{A}_{\kappa}$ can be obtained by its geometric quantization.

It is not difficult to give an example of such one-parameter family of algebras, containing the usual representation of the Poincaré algebra acting on smooth functions (coordinates) on $M_{4}$.

$$
\begin{gathered}
{\left[x^{\mu}, x^{\nu}\right]=i \kappa M^{\mu \nu}} \\
{\left[x^{\lambda}, M^{\mu \nu}\right]=i\left(g^{\lambda \nu} L^{\mu}-g^{\lambda \mu} L^{\nu}\right)} \\
{\left[x^{\mu}, L^{\nu}\right]=i \kappa M^{\mu \nu}} \\
{\left[M^{\lambda \rho}, M^{\mu \nu}\right]=i\left(g^{\lambda \nu} M^{\mu \rho}-g^{\rho \nu} M^{\mu \lambda}+g^{\rho \mu} M^{\nu \lambda}-g^{\lambda \mu} M^{\nu \rho}\right)} \\
{\left[L^{\lambda}, M^{\mu \nu}\right]=i\left(g^{\lambda \nu} L^{\mu}-g^{\lambda \mu} L^{\nu}\right)} \\
{\left[L^{\mu}, L^{\nu}\right]=i \kappa M^{\mu \nu}}
\end{gathered}
$$

where $g^{\mu \nu}$ denotes the Minkowskian metric diag $(-1,1,1,1)$. It follows from the above relations that for $\kappa \neq 0$ the algebras $\mathcal{A}_{\kappa}$ are generated by the $x^{\mu}$. For any value of $\kappa$ there exists an action of the Poincaré group $\mathcal{P}$ on $\mathcal{A}_{\kappa}$ via ${ }^{*}$ automorphisms $(\Lambda, a) \rightarrow \alpha_{(\Lambda, a)}$ defined as:

$\alpha_{(\Lambda, a)} x^{\mu}=\Lambda_{\nu}^{-1 \mu}\left(x^{\nu}-a^{\nu} \mathbf{1}\right), \quad \alpha_{(\Lambda, a)} L^{\mu}=\Lambda_{\nu}^{-1 \mu} L^{\nu}, \quad \alpha_{(\Lambda, a)} I^{\mu \nu}=\Lambda_{\rho}^{-1 \mu} \Lambda_{\sigma}^{-1 \nu} I^{\rho \sigma}$.

The commutation relations between the $I^{\mu \nu}$ and the $L^{\lambda}$ are the relations of the Lie algebra of $S O(4,1)$ if $\kappa$ is positive, of $S O(3,2)$ if $\kappa$ is negative, and of the Poincaré algebra if $\kappa=0$. It follows that the $I^{\mu \nu}$ and the $L^{\lambda}$ generate the corresponding enveloping algebras. The differences of the generators $x^{\mu}-L^{\mu}$ 
are in the center $Z\left(\mathcal{A}_{\kappa}\right)$ of $\mathcal{A}_{\kappa}$; therefore the algebra $\mathcal{A}_{\kappa}$ is the tensor product of the commutative algebra generated by the $\left(x^{\mu}-L^{\mu}\right)$ and the two following Casimir operators:

$$
\begin{gathered}
C_{2}=\kappa g_{\mu \nu} g_{\rho \lambda} I^{\mu \rho} I^{\nu \lambda}+2 g_{\mu \nu} L^{\mu} L^{\nu} \\
C_{4}=g^{\rho \rho^{\prime}}\left(\epsilon_{\rho \lambda m u \nu} L^{\lambda} I^{\mu \nu}\right)\left(\epsilon_{\rho^{\prime} \lambda^{\prime} \mu^{\prime} \nu^{\prime}} L^{\lambda^{\prime}} I^{\mu^{\prime} \nu^{\prime}}\right)
\end{gathered}
$$

where $\epsilon_{\mu \nu \lambda \rho}$ is the totally anti-seymmetric tensor with $\epsilon_{0123}=1$. Therefore also $\mathcal{A}_{0}$ is the tensor product of the commutative akgebra generated by the $\left(x^{\mu}-L^{\mu}\right)$ with the enveloping algebra of the Poincaré Lie algebra generated by the $L^{\mu}$ and the $I^{\mu \nu}$.

It must be stressed here that this Poincaré algebra is not the same as the Poincaré algebra acting on $\mathcal{A}_{0}$ (like on the space-time variables) via the automorphisms $\alpha_{(\Lambda, a)}$; this can be seen also by the fact that $L^{\mu}$ have the dimension of a length. This double appearance of the Poincaré algebra may be interpreted as the necessity to introduce matter besides the space-time itself as soon as we penetrate in the non-commutative sector of the great algebra containing $\mathcal{C}^{\infty}\left(M_{4}\right)$ as a factor.

Since our Casimirs $C_{2}$ and $C_{4}$ are contained in the center of $\mathcal{A}_{\kappa}$, and since they are translationally invariant, we can impose some fixed values on them, thus specifying even more precisely the algebras $\mathcal{A}_{\kappa}$. Since the element $C_{2}$ has the dimension of a length squared, and the element $C_{4}$ that of a length to the power four, the most natural choices amount to attribute the value $\kappa^{2}$ to the element $C_{4}$, while the element $C_{2}$ can be given the following three particular values:

$$
\text { i) } C_{2}=\kappa ; \quad \text { ii) } C_{2}=-\kappa, \quad \text { iii) } C_{2}=0 .
$$

All these choices lead to $g_{\mu \nu} L^{\mu} L^{\nu}=0$ in $\mathcal{A}_{0}^{I}$. Remembering the fact that $\mathcal{A}_{0}^{I}$ has the structure of the enveloping algebra of the Poincaré Lie algebra, the last condition is an analogue of the zero mass condition in the ususal case.

With the value of $C_{4}$ fixed in such a way that the representations found here are all of "zero mass" and "strictly positive spin" type, which gives the algebra $\mathcal{A}_{0}^{I}$ a characteristic two-sheet structure, corresponding to the two possible helicities, which in turn results from the fact that the Lorentz group is not simply connected.

As a concluding remark, we would like to stress the fact that in general the Poincaré covariance of $\mathcal{A}_{\kappa}$ is not necessary; all we need here is to ensure the Poincaré covariance of $\mathcal{A}_{0}$ only. Another deformation of the Poincaré algebra, called "the $\kappa$-Poincaré" has been studied in a series of papers published recently by J. Lukierski and co-authors ([24]).

Their approach is in some sense complementary to the scheme presented above: instead of considering the action of the exact Poincaré group on the space-time containing a non-trivial deformation because of the supposed noncommutative character of the coordinates, one chooses to consider the action of a deformed Poincaré group, called the $\kappa$-Poincaré, on the ordinary space-time. It seems plausible that in the linear limit both these approaches nearly coincide. 


\section{Quantum Spaces and Quantum Groups}

A more radical deformation of usual behaviour of functions describing the coordinates and their differentials consists in modifying the commutation relations not only between the coordinates and their differentials, but also between the coordinates themselves, and between the differentials as well, which would represent a very profound modification of the space-time structure. Moreover, if we look for the transformations that would keep these new relations invariant, we discover that such transformations can not be described by means of ordinary groups, which therefore need to be generalized. Such new generalizations have been introduced by V.Drinfeld, L.Faddeev and S.L.Woronowicz, ([25], [26], $[27])$. and they are known under the name of "Quantum Groups".

The litterature on this subject is very abundant; we shall cite the papers by S.L.Woronowicz [27], as well as the papers of L.C.Biedenharn [28], J.Wess and B.Zumino [29], L.A.Takhtajan [30], V.G.Kac [31]; the list is far from being exhaustive, so that we shall limit ourselves to an outline of the main idea illustrated by a simple example.

Conformally with the spirit of quantum field theories, the most important mathematical object to be studied is the algebra of observables, which are usually functions of few fundamental ones. This approach can be extended to the mathematical study of Lie groups: indeed, we can learn almost everything concerning group's structure from the algebraic structure of functions (real or complex) defined on it.

Consider a compact manifold $G$ which is also a Lie group; let $e$ denote its unit element. The algebra $A$ of functions defined on $G$ has a very particular structure, which is implemented by the following three mappings: $i$ ) for each $f \in A$, there is an element of $A \otimes A$, denoted by $\Delta f$, such that $\Delta f(x, y)=f(x y)$; The mapping $\Delta$ :

$$
A \longrightarrow A \otimes A
$$

is called the coproduct. ii) There exists a natural mapping from $A$ into $\mathbf{C}$ (or R) defined by

$$
\epsilon: f \longrightarrow f(e) \in C
$$

which is called the co-unit iii) There exists a natural mapping of $A$ into itself:

$$
(S f)(x)=f\left(x^{-1}\right)
$$

which is called the antipode

It is easy to see that in the case of the algebra of functions defined on a Lie group the co-product is non-commutative if the Lie group is non-commutative; however, the multiplication law in the algebra $A$ itself remains commutative as long as we consider the functions taking their values in $\mathbf{C}$ or $\mathbf{R}$. This particular structure of an associative commutative algebra $A$ with the three operations defined above, the co-product, the co-unit and the antipode is called the Hopf 
algebra. Now, the natural extension that comes to mind is to abandon the postulate of the commutativity of the product in $A$; in this case, the structure is named the Quantum Group. It should be stressed that a quantum group is not a group, but a general algebra which only in the commutative case behaves as the algebra of functions defined on a Lie group.

One of the most interesting aspects of this theory is the fact that the quantum goups arise quite naturally as the transformations of non-commutative geometries known under the name of quantum spaces introduced by Yu.Manin, J.Wess and B.Zumino, and others. We shall illustrate how a quantum group can be constructed on a simple example in two dimensions called the Manin plane ([32]).

Consider two "coordinates" $x$ and $y$ spanning a linear space and satisfying

$$
x y=q \quad y x
$$

with a complex parameter q different from 1 . Consider a transformation

$$
x^{\prime}=a \quad x+b \quad y, \quad y^{\prime}=c \quad x+d \quad y .
$$

which preserves the relation $x y=\mathrm{q} y x$, i.e. such that

$$
x^{\prime} y^{\prime}=q \quad y^{\prime} x^{\prime}
$$

We shall suppose that the quantities $a, b, c, d$ commute with the "coordinates" $x, y$; the simplest realization of this requirement is achieved by assuming (disregarding the nature of the entries of the matrix) that the multiplication of $x$ by $a, b$, etc. is tensorial, i.e. when we set by definition

$$
x^{\prime}=a \otimes x+b \otimes y .
$$

Then the conservation of the q-commutation relations between $x$ and $y$ leads to the following rules for $a, b, c$ and $d$ :

$$
a c=q \quad c a, \quad b d=q \quad d b, \quad a d=d a+q \quad c b-\left(\frac{1}{q}\right) b c
$$

In order to fix all possible binary relations between the coefiicients $a, b, c$ and $d$ we need three extra relations, which would define $b c, a b$ and $c d$. Such relations can be obtained if we define the "differentials"

$$
\xi=d x, \quad \eta=d y, \quad \xi^{2}=0, \quad \eta^{2}=0 .
$$

satisfying twisted $p$-commutation relations

$$
\xi \eta+\left(\frac{1}{p}\right) \eta \xi=0
$$

with a new complex parameter $p$. Assuming that the exterior differentiation commutes with the transformation matrix and requiring the same relations for $\xi^{\prime}$ and $\eta^{\prime}$, we get

$$
b c=\left(\frac{q}{p}\right) c b, \quad a b=p \quad b a, \quad c d=p \quad d c .
$$


With these relations the matrix algebra defined above becomes associative and can be given the structure of a Hopf algebra as follows:

$$
\Delta\left(\begin{array}{ll}
a & b \\
c & d
\end{array}\right)=\left(\begin{array}{ll}
a \otimes a+b \otimes c & a \otimes b+b \otimes d \\
c \otimes a+d \otimes c & c \otimes b+d \otimes d
\end{array}\right)
$$

and the co-unit as

$$
\epsilon\left(\begin{array}{ll}
a & b \\
c & d
\end{array}\right)=\left(\begin{array}{ll}
1 & 0 \\
0 & 1
\end{array}\right)
$$

The antipode $S$ of a quantum matrix should be defined as its inverse. In order to make such a definition operational, we need a non-commutative generalization of the determinant of a matrix. Such a " $(q, p)$-determinant" should be defined as the combination of parameters appearing in the transformation law for the "elementary area element", i.e. the exterior product of the differentials $\xi$ and $\eta$ :

$$
\xi^{\prime} \eta^{\prime}=D_{q} \xi \eta
$$

which yields immediately

$$
D_{q}=a d-p b c=d a-\left(\frac{1}{q}\right) b c
$$

The determinant $D_{q}$ commutes with $a$ and $d$, but has non-trivial commutation relations with the off-diagonal elements $a$ and $b$ (in what follows, we shall omit the subscript $q$ for the sake of simplicity) :

$$
D b=\left(\frac{p}{q}\right) b D, \quad D c=\left(\frac{q}{p}\right) c D .
$$

It should also possess an inverse $D^{-1}$, which in fact is a new element extending the algebra, and satisfying

$$
D^{-1} D=1=D D^{-1}
$$

Applying these identities to the commutation relations verified by $D$, one finds easily that $D^{-1}$ commutes with $a$ and $b$, and satisfies

$$
b D^{-1}=\left(\frac{p}{q}\right) D^{-1} b, \quad c D^{-1}=\left(\frac{q}{p}\right) D^{-1} c
$$

It is easy to see that

$$
\Delta(D)=D \otimes D, \quad \Delta(D) \Delta\left(D^{-1}\right)=\Delta(1)=1 \otimes 1
$$

and

$$
\Delta\left(D^{-1}\right)=D^{-1} \otimes D^{-1}
$$

The antipode of any matrix can be determined now as follows:

$$
S\left(\left(\begin{array}{ll}
a & b \\
c & d
\end{array}\right)\right)=D^{-1}\left(\begin{array}{cc}
d & \left(\frac{-1}{q}\right) b \\
-q c & a
\end{array}\right)=\left(\begin{array}{cc}
d & \left(\frac{1}{p}\right) b \\
-p c & a
\end{array}\right) D^{-1}
$$


Also

$$
S(D)=D^{-1}, \quad S\left(D^{-1}\right)=S(D)
$$

but $S^{2} \neq 1$. The inverse of the antipode mapping can be also defined as

$$
S\left(\left(\begin{array}{ll}
a & b \\
c & d
\end{array}\right)\right)=D\left(\begin{array}{cc}
a & p q b \\
\left(\frac{1}{p q}\right) c & d
\end{array}\right)
$$

The algebra generated by the matrices defined above, whose entries $a, b, c$ and $d$ satisfy the $(q, p)$-commutation relations is a Hopf algebra; it is denoted by $G L_{p, q}(2, \mathbf{C})$.

A differential calculus on such algebras has been developed by S.L.Woronowicz; the notion of covariant differentiation, if it can be introduced properly, may lead to new and rich extensions of the ideas of connections, curvatures and gauge fields. Here we shall give an example of the realization of covariant derivation and the curvature 2-form on the quantum plane introduced above. These results belong to M. Dubois-Violette et al., published in ([33]).

The algebra of forms on the quantum plane is generated by four elements, $x, y, \xi=d x$ and $\eta=d y$, with the following commutation relations:

$$
\begin{gathered}
x y=q y x, \\
x \xi=q^{2} \xi x, \quad x \eta=q \eta x+\left(q^{2}-1\right) \xi y, \quad y \xi=q \xi y, \quad y \eta=q^{2} \eta y, \\
\xi^{2}=0, \quad \eta^{2}=0, \quad \eta \xi+q \xi \eta=0
\end{gathered}
$$

where $q$ is supposed not to be a root of unity. In (still hypothetical !) future physcical applications the value of the parameter $q$ is supposed to be very close to 1 , and in the linear approximation can be written as $1+\kappa$. The above conditions are of course compatible with the definitions $\xi=d x, \eta=d y$ and with the Leibniz rule, i.e. if we apply the operation $d$ to the first constitutive identity $x y=q y x$, we obtain a relation which is a direct consequence of the four constitutive relations between $x, y$ and their differentials $\xi, \eta$, and so forth.

All the relations between the variables $x, y$ and their differentials $\xi, \eta$ can be written in a more uniform way using a matrix notation which introduces the tensorial product of linear spaces spanned by both $x, y$ and $\xi, \eta$ variables. Denoting $x$ and $y$ by $x^{i}$ and $\xi$ and $\eta$ by $\xi^{k}$, with $i, k=1,2$, we can write

$$
\begin{gathered}
x^{i} x^{j}-q^{-1} \hat{R}_{k l}^{i j} x^{k} x^{l}, \\
x^{i} \xi^{j}-q \hat{R}_{k l}^{i j} \xi^{k} x^{l}, \\
\xi^{i} \xi^{j}+q \hat{R}_{k l}^{i j} \xi^{k} \xi^{l} .
\end{gathered}
$$

The tensor product of two 2-dimensional spaces is 4-dimensional, but the indices that are grouped two by two can be re-labeled with their values ranging from 1 to 4 , and the $R$-matrix can be written as an ordinary $4 \times 4$ matrix:

$$
\hat{R}=\left(\begin{array}{cccc}
q & 0 & 0 & 0 \\
0 & \left(q-q^{-1}\right) & 1 & 0 \\
0 & 1 & 0 & 0 \\
0 & 0 & 0 & q
\end{array}\right)
$$


If the $S L_{q}(2, \mathbf{C})$ matrix (corresponding to the case $p=q^{-1}$ in the more general notation $G L_{q}(p, q)(2, \mathbf{C})$ introduced above) is written, with the same indices $k, l=1,2$ as

$$
a_{k}^{i}=\left(\begin{array}{ll}
a & b \\
c & d
\end{array}\right)
$$

then the invariance of the $q$-commutation relations with respect to the simultaneous transformation of the linear spaces $x, y$ and $\xi, \eta$ by a matrix belonging to the quantum group $S L_{q}(2, \mathbf{C})$ amounts to the following relation:

$$
\hat{R}_{k l}^{i j} a_{m}^{k} a_{n}^{l}=a_{k}^{i} a_{l}^{j} \hat{R}_{m n}^{k l}
$$

If we extend trivially the action of the differential $d$ onto the quantum group $S L_{q}(2, \mathbf{C})$ itself by requiring all the coefficients $a_{k}^{i}$ to be constant,

$$
d a_{k}^{i}=0,
$$

The coaction of $S L_{q}(2, \mathbf{C})$ on the $x^{i}$ and the $\xi^{k}$ can be defined then as follows:

$$
\tilde{x}^{i}=a_{k}^{i} \otimes x^{k}, \quad \tilde{\xi}^{j}=a_{m}^{j} \otimes \xi^{m} .
$$

It can be found without much pain that the new variables $\tilde{x}^{i}$ and $\tilde{\xi}^{k}$ satisfy the same twisted commutation relations as formerly $x^{i}$ and $\xi^{k}$.

As in the case of the matrix model of non-commutative geometry, one can introduce a canonical 1-form by defining

$$
\theta=x \eta-q y \xi, \quad \text { satisfying } \quad \theta^{2}=0 .
$$

and is invariant under the coaction of $S L_{q}(2, \mathbf{C})$ with $\tilde{\theta}=\mathbf{1} \otimes \theta$ and has the following commutation relations with the variables $x^{k}, \xi^{m}$ :

$$
x^{k} \theta=q \theta x^{k} ; \quad \xi^{m} \theta=-q^{3} \theta \xi^{m}
$$

Up to a complex multiplicative constant this is the unique element of $\Omega^{1}$ (the space of $q$-one forms) verifying the above properties.

To define covariant derivation, we must introduce first the permutation operator $\sigma$ mapping the tensor product $\Omega^{1} \otimes_{\mathcal{A}} \Omega^{1}$ into itself. As a matter of fact, the operator $\sigma$ turns out to be just the inverse of the matrix $q \tilde{R}_{k l}^{i j}$. We can write it down using the explicit indices $i, j, .$. as follows:

$$
\begin{gathered}
\sigma(\xi \otimes \xi)=q^{-2} \xi \otimes \xi, \quad \sigma(\xi \otimes \eta)=q^{-1} \eta \otimes \xi \\
\sigma(\eta \otimes \xi)=q^{-1} \xi \otimes \eta-\left(1-q^{-2}\right) \eta \otimes \xi, \quad \sigma(\eta \otimes \eta)=q^{-2} \eta \otimes \eta
\end{gathered}
$$

as well as

$$
\begin{gathered}
\sigma(\xi \otimes \theta)=q^{-3} \theta \otimes \xi, \quad \sigma(\theta \otimes \xi)=q \xi \otimes \theta-\left(1-q^{-1}\right) \theta \otimes \xi, \\
\sigma(\eta \otimes \theta)=q^{-3} \theta \eta, \quad \sigma(\theta \otimes \eta)=q \eta \otimes \theta-\left(1-q^{-2}\right) \theta \otimes \eta .
\end{gathered}
$$


and also

$$
\sigma(\theta \otimes \theta)=q^{-2} \theta \otimes \theta
$$

If we suppose that $q^{2} \neq-1$, then the exterior algebra is obtained by dividing the tensor algebra over $\Omega^{1}$ by the ideal generated by the three eigenvectors :

$$
\xi \otimes \xi, \quad \eta \otimes \eta \quad \text { and } \quad \eta \otimes \xi+q \xi \otimes \eta,
$$

corresponding to the eigenvalue $q^{-2}$.

The symmetric algebra of forms is obtained by dividing the tensor algebra over $\Omega^{1}$ by the ideal generated by the eigenvector $\xi \otimes \eta-q \eta \otimes \xi$ corresponding to the eigenvalue -1 .

There is a unique one-parameter family of covariant derivatives compatible with the algebraic structure of the algebra of forms defined above. It is given by

$$
D \xi^{k}=l^{-4} x^{k} \theta \otimes \theta
$$

where the parameter $l$ must have the dimension of a length. From the invariance of $\theta$ it follows that $D$ is invariant under the coaction of $S L_{q}(2, \mathbf{C})$. The analog of torsion vanishes identically.

Finally, the analog of the curvature tensor can be defined here as

$$
D^{2} \xi^{k}=\Omega^{k} \otimes \theta=-\Omega_{j}^{k} \otimes \xi^{l}
$$

with the curvature 2 -forms given by the following matrix:

$$
\Omega_{j}^{i}=l^{-4}\left(1+q^{-2}\right)\left(1+q^{-4}\right)\left(\begin{array}{cc}
q^{2} x y & -q x^{2} \\
q^{2} y^{2} & -x y
\end{array}\right) \xi \eta
$$

It vanishes for the particular values of $q$, namely, when $q= \pm i$ or $q^{2}= \pm i$, but is different from zero when $q=1$. The Bianchi identity is trivially satisfied.

No metric structure compatible with this structure can be introduced except for the trivial case when $q=1$.

\section{Conclusion}

We tried to present here a few versions of non-commutative generalizations of differential geometry which are believed to serve - hopefully in some foreseable future - as new mathematical tools that will help us to describe the effects of quantum gravity. Frankly speaking, in spite of beauty and sophistication of certain models, it is hard to share this belief.

It does not mean that our efforts should be reduced or stopped at once. "Ars longa, vita brevis" , and there is still a lot of time ahead, especially as compared to the cosmological scale. The overall impression might be pessimistic, but there is always plenty of things to do.

For example, if we look at the diagram of Sect.1, we can note that besides the "Relativistic Quantum Field Theory" there is another unexplored corner, the "Non-Relativistic Quantum Gravity". Maybe we should pay some more attention to this direction, too? Or at least, if such a theory can not be formulated, try to give valuable reasons why this is the unique combination of limits of fundamental constants that can not be realized as a coherent theory? 


\section{References}

1. J.E. Moyal, Quantum mechanics as a statistical theory, Proc.

Camb.Phil.Soc., 45, 99 (1949)

2. P.A.M. Dirac, The Fundamental Equations of Quantum Mechanics, Proc.Roy.Soc., A 109, p.642 (1926); also On Quantum Algebras, Proc. Cambridge Phil.Soc., 23 p.412 (1926)

3. P.A.M. Dirac, as cited in The Mathematical Intelligencer 11 p.58 (1989)

4. R. Arnowitt, S. Deser, C. Misner, in Recent Developments in General Relativity, ed. L. Witten, Wiley, London- New York, (1962)

5. B. DeWitt, Physical Review, 160, p.1113 (1963)

6. J.M. Souriau, Structure des Systèmes Dynamiques, Dunod, Paris, (1969)

7. D. Simms, in Differential Geometrical Methors in Math. Physics, Springer Lecture Notes in Mathematics, 170, ed. K. Bleuler and A.Reetz, Springer-Verlag, (1970).

8. B. Kostant, Quantization and Unitary Representations, Lecture Notes in Mathematics, 170, Springer Verlag p.237 (1970).

9. J. von Neumann, Mathematical Foundations of Quantum Mechanics, Princeton University Press, (1955)

10. A. Ashtekar, in Mathematical Physics towards the 21-st century, R. Sen and A. Gersten eds., Ben Gurion University of the Neguev Press, p 230 (1994).

11. C. Rovelli, Nucl. Physics B 405, p.797 (1993), also the lectures in this volume.

12. D. Quillen, Topology 24, p. 89 (1985)

13. A. Connes, Non-Commutative Geometry, Acad. Press., New York (1994)

14. M. Dubois-Violette, C.R.Acad.Sci. Paris, 307, Sér.I, p.403 (1989)

15. M. Dubois-Violette, R. Kerner and J. Madore, Journ. of Math. Physics, 31 (2), p. 316 (1990)

16. M. Dubois-Violette, R. Kerner and J. Madore, ibid, p. 323 (1990)

17. A. Connes and J. Lott, Nucl. Phys. Proc. Suppl. B 18, p.29 (1990)

18. R. Coquereaux, G. Esposito-Farèse and J. Vaillant, Nucl. Phys B, 353, p.689 (1991)

19. A. Chamseddine, G. Felder and J. Frölich, Commun. Math. Physics, 155, p. 205 (1993) 
20. J. Madore, T. Masson and J. Mourad, Class. and Quantum Gravity, 12, p. 1429 (1995)

21. M. Dubois-Violette, J. Madore and R. Kerner, Journal of Math. Physics, 39, No 2, p. 730 (1998)

22. S. Doplicher, K. Friedenhagen and J.E. Roberts, Comm. Math. Phys., 172, p. 187 (1995).

23. F. Bayen, M. Flato, C. Fronsdal, A. Lichnerowicz and D. Sternheimer, Ann. Phys. (N.Y.) 111 p. 61, p. 111 (1978)

24. J. Lukierski, A. Nowicki and H. Ruegg, Phys. Lett. B 293, p.419 (1992)

25. V.G. Drinfeld, Quantum Groups, in Proceedings of Intern. Congress of Mathematics, Berkeley, p.798

26. L.D. Faddeev, Proc. of Schladming Winter School, p.89 (1989)

27. S.L. Woronowicz, Communications in Math. Phys. 111 p. 613 (1987) also: P. Podleś and S.L. Woronowicz, ibid, 178, p.61 (1996)

28. L. Biedenharn, Quantum Groups, Lecture Notes in Physics, 370, H.D. Doebner and J.D. Hennig eds., Springer-Verlag, p.67 (1990)

29. J. Wess and B. Zumino, Nucl. Phys. B (Proc. Suppl.) 18, p.32 (1990)

30. L.A. Takhtajan, Adv. Studies in Pure and Applied Mathematics, 19, p.435 (1989)

31. V.G. Kac, Colloque Dixmier, p. 471, Progrss in Mathematics, Vol. 92 Birkhäuser, Boston (1990); see also : C. de Concini and V.G. Kac, J. Am. Math. Soc. 5 (1), p. 151 (1992).

32. Yu. Manin, Commun. Math. Physics, 123, p.163 (1989)

33. M. Dubois-Violette, J. Madore, T. Masson and J. Mourad, Lett. in Math.Physics, 35, p. 351 (1995). 